\title{
Laparoscopic approach to hysterectomy
}

Hakan Nazik ${ }^{1}$

${ }^{1}$ M.D., Adana Numune Education and Research Hospital, Department of Obstetrics and Gynecology, Adana, Turkey

\section{Abstract}

Modern laparoscopic surgery is widely used throughout the world as it offers greater advantages than open procedures. The laparoscopic approach to hysterectomy has evolved over the last 20 years. Hysterectomies are performed abdominally, vaginally, laparoscopically or, more recently, with robotic assistance. Indications for a total laparoscopic hysterectomy are similar to those for total abdominal hysterectomy, and most commonly include uterine leiomyomata, pelvic organ prolapse, and abnormal uterine bleeding. When hysterectomy is going to be performed, the surgeon should decide which method is safer and more cost-effective. This paper aims to make a review of the indications, techniques and advantages of laparoscopic hysterectomy as well as the criteria to be used for appropriate patient selection.

\section{Keywords}

Laparoscopic hysterectomy, Indications, Technique, Complication 
- Diagnostic laparoscopy with vaginal hysterectomy (VH)

- Laparoscopic vault suspension after vaginal hysterectomy

- Laparoscopic assisted vaginal hysterectomy (LAVH)

- Laparoscopic hysterectomy (LH)

- Total laparoscopic hysterectomy (TLH)

- Laparoscopic supracervical hysterectomy (LSH) or Classic abdominal SEMM hysterectomy (CASH), where SEMM stands for Serrated Edge Macro Morcellator

- Laparoscopic hysterectomy with lymphadenectomy (LHL)

- Laparoscopic hysterectomy with lymphadenectomy and omentectomy $(\mathrm{LHL}+0)$

- Radical laparoscopic hysterectomy (RLH), which is usually the combination of a laparoscopic lymphadenectomy combined with a radical Shauta's vaginal hysterectomy

Table 1. Classification of laparoscopic

hysterectomy. Modified from [5]

\section{Introduction}

Modern laparoscopic surgery is widely used throughout the world as it offers greater advantages than open procedures. In the United States, hysterectomy is the most common surgical procedure performed after pregnancy-related surgical procedures, of which over $90 \%$ were performed for benign conditions [1]. The laparoscopic approach to hysterectomy has evolved over the last 20 years [2,3]. Hysterectomies are performed abdominally, vaginally, laparoscopically or, more recently, with robotic assistance. Total laparoscopic hysterectomy (TLH) is defined by the laparoscopic ligation of the ovarian

arteries and veins with the removal of the uterus vaginally or abdominally, along with laparoscopic closure of the vaginal cuff. If laparoscopy is only used for visualizing the pelvis, freeing adhesions, dividing ligaments, or developing spaces, then the procedure is termed a laparoscopic assisted vaginal hysterectomy (LAVH). A common classification system is shown in the Table I [4].

When hysterectomy is going to be performed, the surgeon should decide which method is safer and more cost-effective. A review of the current literature shows that vaginal hysterectomy offers improved results and lower complication rates compared to laparoscopic or abdominal hysterectomy [6]. When compared with abdominal hysterectomy, better visualization of pelvic anatomy and less blood loss, wound infections, and postoperative pain as well as shortened length of stay and faster resumption of normal activities could be considered as the most important advantages of TLH [7]. The introduction of uterine manipulators made the uterine vessels, vagina and rectum accessible from multiple angles. Where performing vaginal hysterectomy would be inappropriate, the surgeon should decide which surgical method to use: laparoscopic hysterectomy, robotic-assisted hysterectomy or abdominal hysterectomy. This paper aims to make a review of the indications, techniques and advantages of laparoscopic hysterectomy as well as the criteria to be used for appropriate patient selection.

\section{Total laparoscopic hysterectomy}

\section{Indications}

Indications for a TLH are similar to those for total abdominal hysterectomy, and most commonly include uterine leiomyomata, pelvic organ prolapse, and abnormal uterine bleeding. The American College of Obstetricians and Gynecologists has listed the following as potential indications for laparoscopic assistance to facilitate vaginal hysterectomy: need for adhesiolysis, need for treatment of endometriosis, need for management of large leiomyoma(s) to facilitate uterine extraction, and the need for ligation of the infundibulopelvic ligaments to facilitate oophorectomy [8]. TLH may also be indicated for resection and debulking in both malignant and premalignant diseases [9]. Laparoscopy may be used to remove the uterus via the vaginal route where vaginal hysterectomy is difficult to perform [10]. In laparoscopic supracervical hysterectomy or TLH, large uteri and giant myomas can be removed through laparoscopic incisions by mechanical morcellation [11]. Dissection of the rectovaginal septum and uterosacral ligaments may be difficult during vaginal hysterectomy in deep infiltrating endometriosis. It may even be impossible to remove many lesions via the vaginal route. Laparoscopy is more 
advantageous in that it offers the ability to better visualize the tissue and remove deeply infiltrating endometriotic foci. Highly experienced surgeons can remove the ovaries via the vagina, but this is a difficult operation for most surgeons. However, many surgeons are more experienced in laparoscopic oophorectomy [12]. Therefore, laparoscopic route is the preferred route for hysterectomy where the patients undergo oophorectomy.

\section{Classification}

TLH is the conduct of the entire surgical procedure laparoscopically and the removal of the uterus via the vaginal route or by morcellation. However, morcellation is not performed if there is suspicion of uterine cancer [13]. After removal of the uterus, the vaginal cuff is closed using laparoscopic suturing techniques. Laparoscopic-assisted vaginal hysterectomy is a method to provide laparoscopic assistance to vaginal hysterectomy. The completion of the remaining procedure via the vaginal route after the division of the upper vascular pedicles and the parametria are performed laparoscopically. Following completion of the procedure, it allows abdominal bleeding control by reinsufflation. Robotic-assisted laparoscopic hysterectomy is more costly than conventional laparoscopic surgery and outcomes appear comparable to conventional laparoscopic or vaginal hysterectomy [14]. Comparative studies have found that complication rates are similar for conventional and robotic-assisted laparoscopic hysterectomy [15]. Single port laparoscopy, or laparoendoscopic single-site surgery (LESS), is one of the latest innovations in laparoscopy. The first LESS total hysterectomy was reported in 2010 [16]. LESS can be used for total laparoscopic hysterectomy or LESS-assisted vaginal hysterectomy. Laparoscopic radical hysterectomy can be either fully performed laparoscopically or completed by radical vaginal hysterectomy after lymphadenectomy and the dissection of upper pedicles [17].

\section{Contraindications}

Laparoscopic hysterectomy has few contraindications. The most important contraindication is the laparoscopic surgeon lacking in experience and knowledge in laparoscopic hysterectomy [18]. Causes including large uterus, giant myomas, and a history of surgical or deeply infiltrating endometriosis may result in contraindications both because they make abdominal access more difficult and they do not allow for adequate abdominal working space due to adhesion [19]. Cases where abdominal access becomes impossible due to morbid obesity, and where the patient is unable to tolerate anesthesia in the lithotomy position due to a medical condition are other causes.

\section{Equipment and setup}

A well-trained endoscopic surgical team, sufficient equipment, surgical instruments and a well-organized operating room are needed for successful and effective endoscopic surgeries [20]. Therefore, the surgeon who will perform laparoscopic surgery should personally check the entire operating room setup, the patient's position and all laparoscopic equipment. The patient should be placed in the modified dorsal lithotomy position, and the patient's arms should be properly secured at the sides. This will help the surgeon to be able to approach the patient's pelvis at a suitable angle at both sides, allowing for the surgeon's movements to the patient's shoulders and head [20]. Shoulder holders or pads are routinely used to protect the patient in the 20-30 ${ }^{\circ}$ Trendelenburg position which is required during gynecological laparoscopy. Care should be taken to hold the patient's legs in a downward direction in the modified dorsal lithotomy position. Otherwise, the surgeon may find it difficult to approach to the upper abdomen. Placement of the uterine manipulator requires a bivalve speculum or vaginal retractors, a tenaculum, and cervical dilators. To achieve safe and successful procedural outcomes, the uterine manipulators used in laparoscopic hysterectomy should be capable of performing many different tasks. Their most obvious function is the mobilization of the uterus by allowing movement in three 


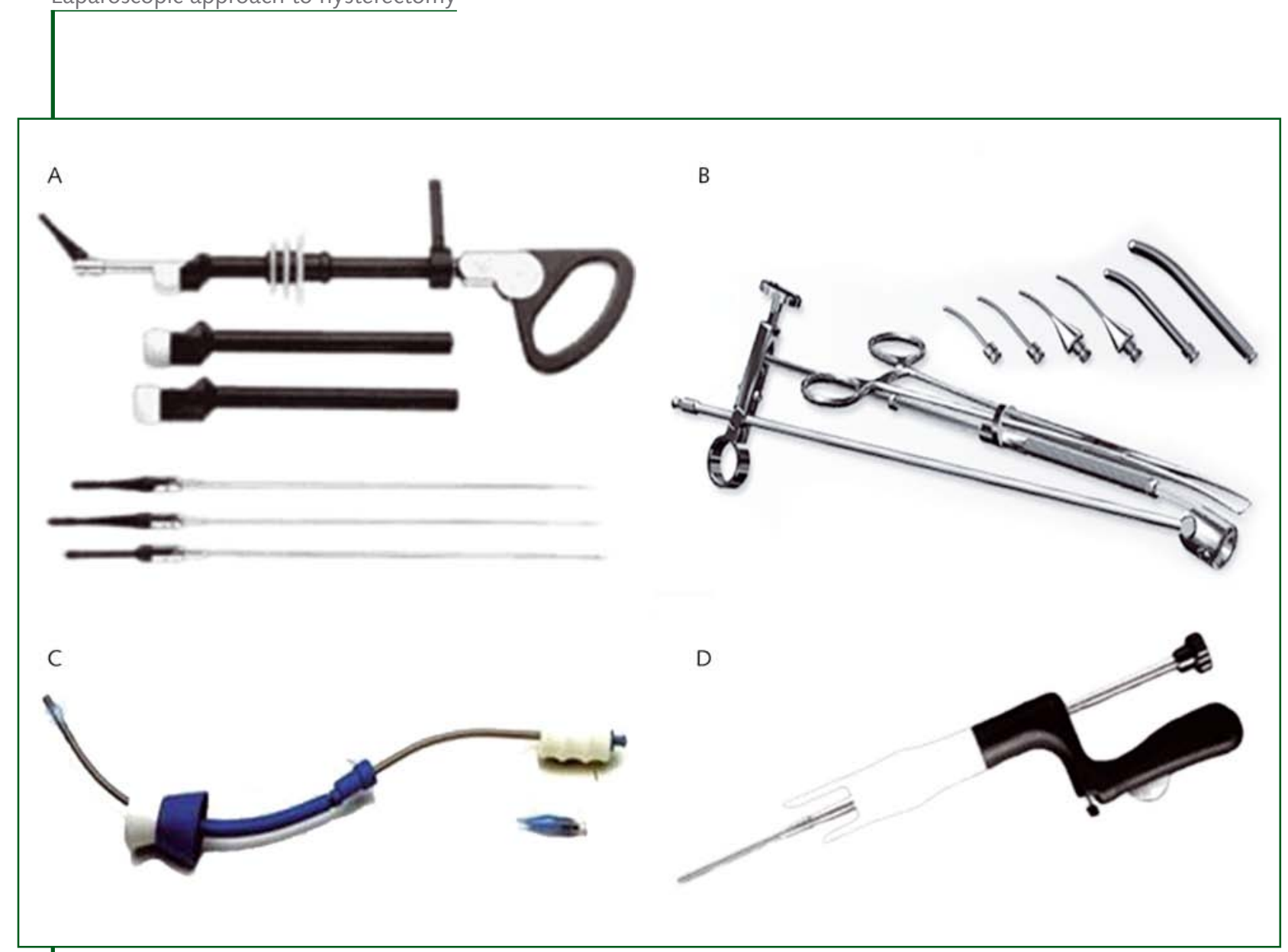

Figure 1. Uterine manipulators: Clermond-Ferrand Uterine Manipulator (A), Valtchev Uterine Mobilizer (B), Vcare Uterine Manipulator (C), HOURCABIE Vaginal Fornices Presenter (D)

positions: anteversion and retroversion, lateral and elevated. Today, the Vcare Uterine Manipulator/ Elevator (ConMed Corporation, Utica, N.Y.), the Clermond-Ferrand Uterine Manipulator (Model K.Storz Endoskope,Tuttlingen, Germany), the Valtchev Uterine Mobilizer (Conkin Surgical, Toronto, Canada), and the HOURCABIE Vaginal Fornices Presenter are some examples of the most commonly used manipulators in laparoscopic hysterectomy (Figure 1).

Typically, the surgeon is positioned to the left, and the assistant stands to the right of the patient facing the patient's legs. The second assistant holds the uterine manipulator between the patient's legs. Although every clinic has its own unique operating room setup, the set-up of a typical operating room is given in the following Figure 2.

The assistant surgeon holds the camera in one hand while he generally holds atraumatic, Maryland, or bowel graspers in his other hand to assist with the retraction. The surgeon uses the laparoscopic instruments with both hands. Monopolar, bipolar, harmonic, or sealing devices are used during the operation to coagulate the vessels and cut the tissue. In laparoscopic hysterectomy, suction irrigators are important and widely-used instruments.

It is important that the surgeon is informed about the equipment and applications used in laparoscopy. This will help him to easily cope with potential technical problems during the operation. Many surgical tools and energy sources are available to coagulate, dissect, and reapproximate the tissue via laparoscopy. Suture materials used in routine operations are also used in laparoscopic surgeries with needle drivers. There are also suture materials such as barbed sutures which allow knotless suturing [21]. Various electrosurgical instruments are used to coagulate and dissect the tissues. Monopolar electrosurgery is a very effective and widely-used energy modality in the dissection of tissues and sealing 


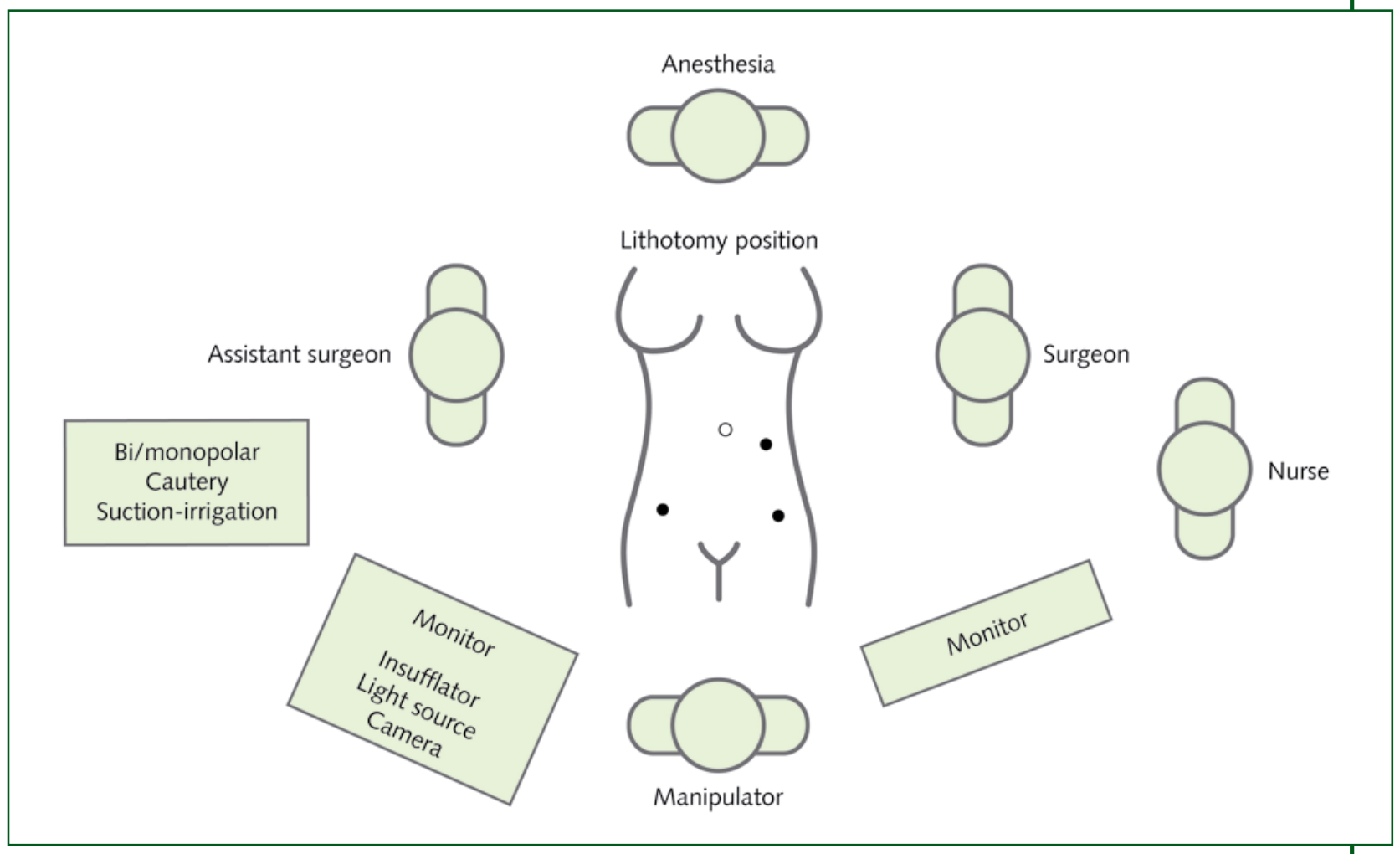

Figure 2. Operating room setup

of small vessels [22]. Its most important disadvantage is the risk of energy transfer and burning of non-targeted tissues in low-resistance tissues rich in fluids and electrolytes. In bipolar electrosurgery, electrical energy is transmitted between both ends of the bipolar coagulator and transforms into a source of energy. This eliminates the risk of burning of non-targeted tissues. However, bipolar electrosurgery generates an important amount of heat in the setting and the whitened tissue border leads to irreversible necrosis [23]. Rinsing of the surgical instruments is another aspect to be taken into consideration, when autoclave sterilization is not used. Chemical artefacts may cause inadvertent demage to the viscera [24]. So, it has to be used in consideration of thermal spread. The ultrasonic scalpel operates by means of a blade that vibrates longitudinally at a frequency of $55.5 \mathrm{mHz}$. The end of the device oscillates at about 55,000 cycles per second, which results in precise cutting, generating neither smoke nor char. The devices place three rows of titanium staples across the pedicle and a trigger activates a sharp blade, which cuts in between them [25]. The most recent vessel sealing devices are the Ligasure ${ }^{\mathrm{mm}}$ and the Plasma Kinetic ${ }^{\mathrm{mm}}(\mathrm{PK})$ systems. These devices use bipolar enery modality and seal wide-diameter vascular pedicles [26]. Another newly-developed device, the $5 \mathrm{~mm}$ EnSeal ${ }^{\mathrm{m}}$ instrument, provides a tissue-controlled energy deposition, with the "smart electrode" resulting in high seal strengths in vessels ranging in diameter from less than $1 \mathrm{~mm}$ to $7 \mathrm{~mm}$. The carbon dioxide $(\mathrm{CO} 2)$ laser or the Contact Nd:YAG laser and the Argon beam coagulator $(\mathrm{ABC})$ are other energy modalities used in laparoscopy.

\section{Surgical technique}

For a successful laparoscopic hysterectomy surgery, the surgeon should be in the operating room before the patient is anesthesized, check the laparoscopic instruments, the $\mathrm{CO}_{2}$ tube and the insufflator, the light source, and the monitor, and make sure that the patient is appropriately placed in the lithotomy 
position. The patient should be placed in the $20-30^{\circ}$ Trendelenburg position. The patient's arms should be placed at the sides and the surgical team should be positioned as shown in the Figure 2.

The uterine manipulator should be placed before the surgical intervention. Proper placement of the uterine manipulator and its use by an experienced assistant are important factors affecting the success of the overall surgical procedure. Many uterine manipulators are used to serve this purpose (Figure 1). We typically use Clermont-Ferrand uterine manipulators. Disposable manipulators can be used with this purpose, but this increases associated costs.

A intra-umbilical incision is used to introduce pneumoperitoneum. The primary trocar can be inserted via a closed technique either by insufflation using the Veress needle or by direct-trocar entry technique or using the open (Hasson) technique. These techniques have similar complication rates, neither of them being superior to the other [27]. Trocar selection, number, and location may depend on several factors including the patient's habitus, surgical history, uterine or pelvic mass size, indications for oncologic staging, available instruments, and the degree of assistant participation.

Video recording may be started before trocar insertion. In the absence of a history of laparotomy, a $10-12 \mathrm{~mm}$ trocar is placed at the umbilicus. Otherwise, in patients with prior laparotomy, the trocar can be placed in the left-upper quadrant or above the umbilicus after orogastric catheterization. Once placed successfully through the peritoneum, pneumoperitoneum is developed with high flow $\mathrm{CO}_{2}$ at $20 \mathrm{mmHg}$. Once adequate pneumoperitoneum is established, the gas flow is reduced to $15 \mathrm{mmHg}$ and the laparoscope is introduced. Two additional 5- $\mathrm{mm}$ satellite trocars are placed in the each ilioingiunal area, and a third satellite trocar can be placed in the left-upper quadrant as necessary (Figure 2). A conscious effort should always be made to avoid the inferior epigastric vessels and perforating arteries of the anterior abdominal wall during trocar placement. The inferior epigastric arteries can typically be visualized with the laparoscope while the abdomen is palpated from above prior to trocar placement. Transabdominal illumination of the anterior abdominal wall may also facilitate vessel identification and proper trocar placement.The ureter is the organ that is most at-risk during hysterectomy procedures as it lies only one centimeter to the cervix laterally. The second most likely place of injury is at the level of infundibulopelvic ligaments during oophorectomy. It should be identified visually by the characteristic peristalsis, and its course traced down towards if the operator is unable to visualize the ureter directly, then careful dissection should be performed in an attempt to protect this structure.

Hysterectomy starts after the identification of ureter placement. If oophorectomy will be performed, then first the infundibulopelvic ligament (IP) is sealed using suture, bipolar electrosurgery, stapling devices, or vascular clips. If the only hysterectomy is to be performed, the round ligament and the utero-ovarian ligament (UO) are sealed and cut, respectively, and the procedure continues. We presented a laparoscopic hysterectomy operation in the Figure 3. Sealing of the uterine side of the pedicles is recommended to decrease back-bleeding. After the sealing and cutting of the ligament, bladder flap is created using a selected cutting device just below the utero-vesicle fold. The flap is mobilized using sharp and blunt dissection (Figure 3).

After the bladder flap is created at the anterior, uterine arteries are sealed and cut at the sides at the internal cervical os level (Figure 3). At this stage, the surgeon should be close to the uterus in order to prevent ureteral injuries. Having a cyanotic appearance, supracervical hysterectomy can be performed for the uterus at this stage, or the operation can be completed via the vaginal route. For TLH, anterior and posterior culdotomies may be performed using a monopolar cautery (Figure 3). If supracervical hysterectomy is performed, then the uterus has to be taken out through the abdominal ports by morcellation.

After specimen removal, attention is turned to the closure of the vaginal cuff. Several methods and materials are available for intra-abdominal cuff closure. For the introduction of post-TLH pneumoperitoneum, the vagina should be closed using a sterile gloves filled with gauze or by similar methods. Interrupted or continuous sutures may be tied intracorporeally with a needle driver and grasper or 
extracorporeally with a knot pusher and grasper (Figure 3). Moreover, the vagina may be tied up, as in conventional methods, via the vaginal route. In order to prevent enterocele and cuff prolapse, suture can be used to close the cuff with the surrounding pericervical fascial tissue by plicating and shortening the uterosacral tissue in the manner described by McCall [28]. The abdomen should be inspected at the

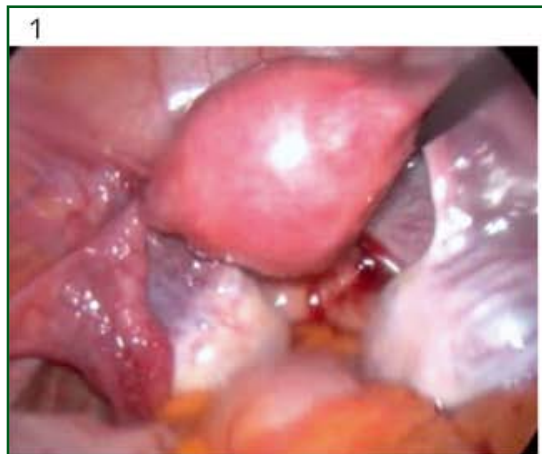

4

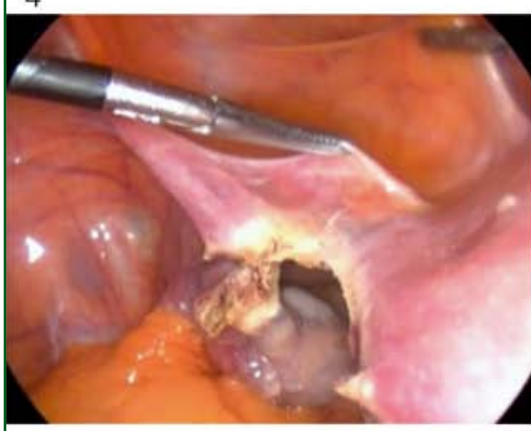

7

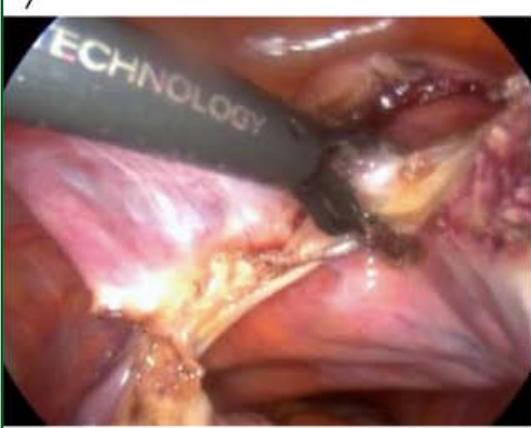

10

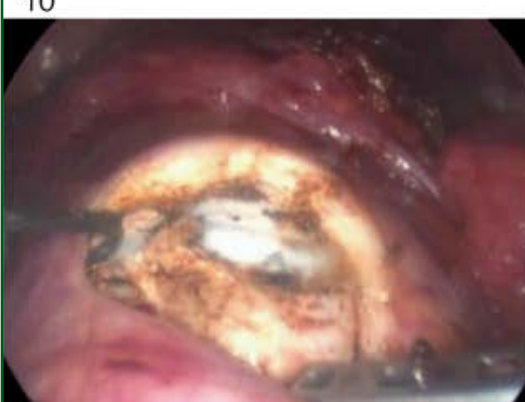

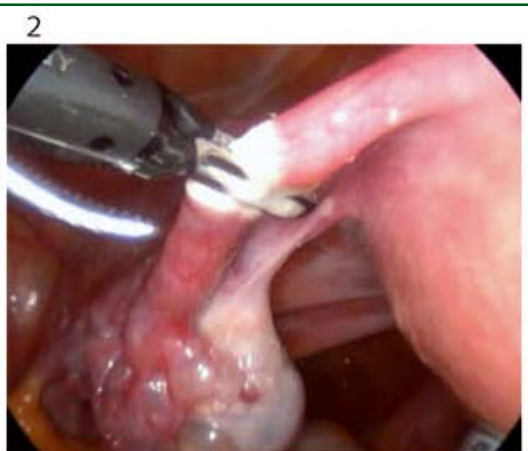

5

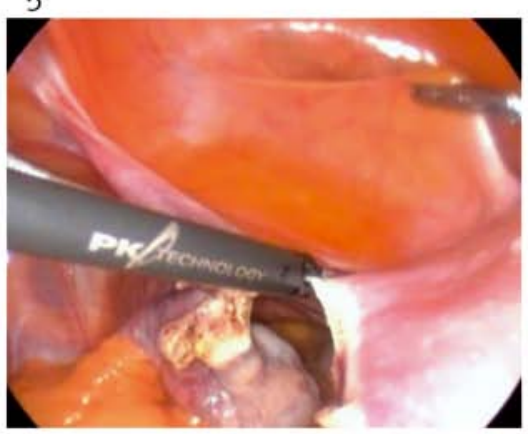

8
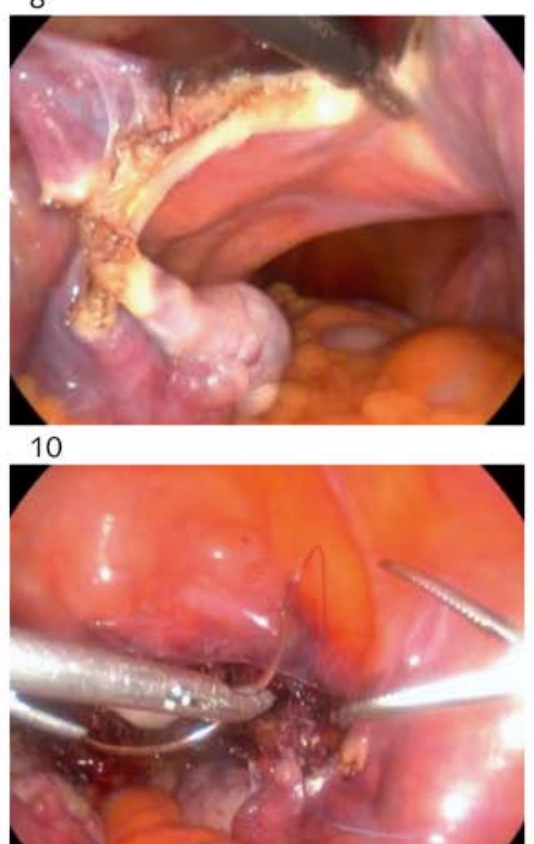

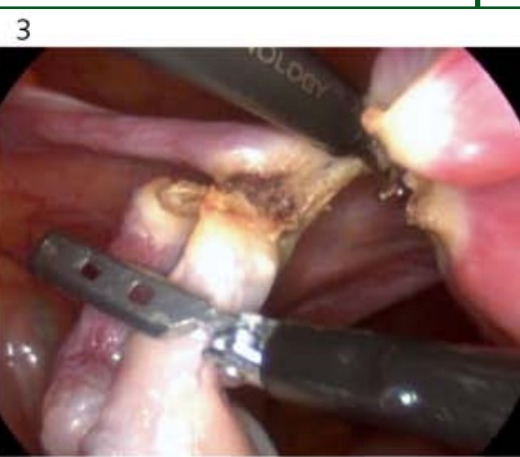

6

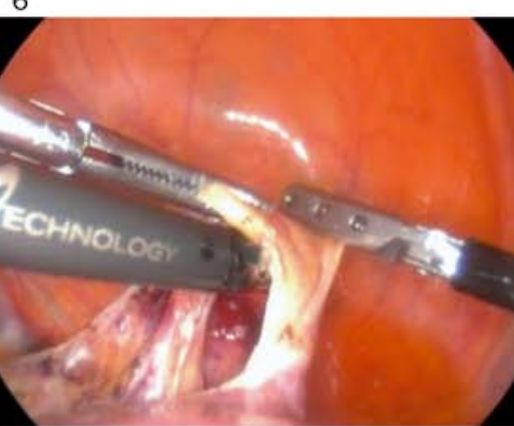

9
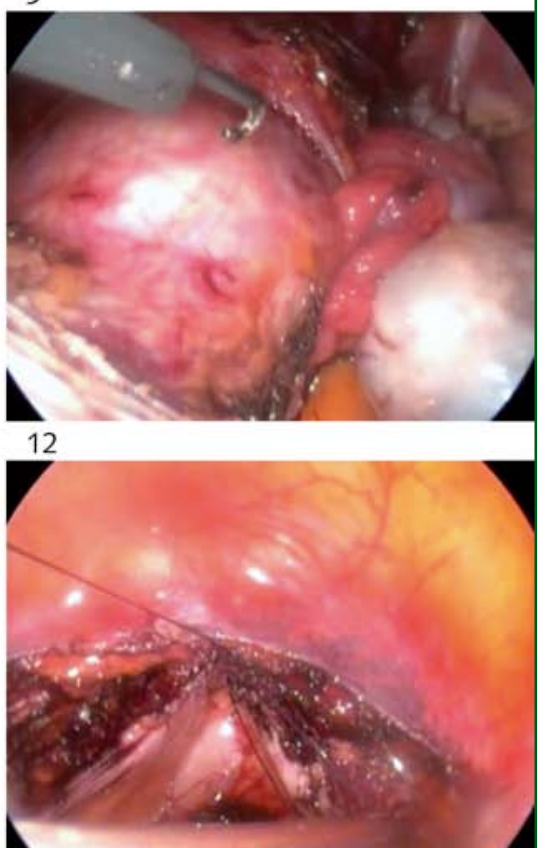

Figure 3. Laparoscopic hysterectomy procedure: pelvic cavity prior to surgery (1), dissection of the Fallopian Tubes (2), dissection of the utero-ovarian ligaments (3), dissection of the anterior leaf of the broad ligament (4), dissection of cardinal ligaments (5), separation of the uterus from the bladder and vagina (6), bilateral occlusion of the uterine vessels (7-8), anterior and posterior colpotomy by using monopolar cautery (9-10), removal of the uterus and closure of the vaginal cuff (11-12) 
Questions for further research

Current literature have found that complication rates are similar for conventional and robotic-assisted laparoscopic hysterectomy. Early studies found prolonged operating times for robot-assisted procedures, but this appears to have been the result of a lack of experience with this new technology. Today robotic asisted laparoscopic hysterectomy is being performed more commonly than the past. Together with decreasing the costs and improvement in technology robots seem to be more commonly used in future and maybe all kinds of hysterectomies will be performed with robots.

completion of the laparoscopic procedure. Intraabdominal pressure should be reduced and bleeding checked. Complete abdominal aspiration and irrigation should be performed, the trocars should be carefully removed from the abdomen, and care should be taken to make sure there is no bleeding. $\mathrm{CO}_{2}$ should be completely discharged to make sure there is no postoperative pain. Fascial closure of the larger 10 or $12 \mathrm{~mm}$ ports is recommended to prevent an incisional hernia.

\section{Complications}

Despite advanced technology and experience, laparoscopic complications remain a major cause of morbidity. The incidence of major and minor complications from laparoscopic procedures ranges from $0.1 \%-10 \%$, with over $50 \%$ of injuries occurring at the time of entry and $20-25 \%$ not recognized until the postoperative period [29]. The rapid increase in the number of procedures being performed, the introduction of new equipment, and variability in the training of surgeons all contribute to the complication rate. It is well known that there is a strong correlation between the complication rate and the surgeon's level of experience. The more experience a surgeon has, the lower the complication rate [30].

The review in brief

Clinical question This review is about the indications, technique and advantages of laparoscopic hysterectomy and how appropriate patient selection should be done.

Type of review Narrative

Search of the Search on PubMed for all the most recent articles on laparoscopic hysterectomy, with keywords:

literature laparoscopic hysterectomy, indications, technique and complication.

Conclusions A review of the current literature shows that vaginal hysterectomy offers improved results and lower complication rates compared to laparoscopic or abdominal hysterectomy. When hysterectomy is going to be performed, the surgeon should decide which method is safer and more cost-effective. Where performing vaginal hysterectomy would be inappropriate, the surgeon should decide which surgical method to use: laparoscopic hysterectomy, robotic-assisted hysterectomy or abdominal hysterectomy. 
3. Reich H, DeCaprio J, McGlynn F. Laparoscopic hysterectomy. J Gynecol Surg 1989; 5: 213; http:// dx.doi.org/10.1089/gyn.1989.5.213

4. Johns DA. Laparoscopic Assisted Vaginal Hysterectomy. In: Sutton CJG, Diamond MP (Eds.). Endoscopic Surgery for Gynaecologists, 2nd Ed. London: WB Saunders, 1998; p. 300

5. Garry R, Reich H. Basic techniques for advanced laparoscopic surgery. In: Garry R, Reich H (Eds). Laparoscopic Hysterectomy. Oxford: Blackwell Scientific, 1992; p. 46

6. ACOG Committee Opinion No. 444: choosing the route of hysterectomy for benign disease. $\mathrm{Ob}$ stet Gynecol 2009; 114: 1156; http://dx.doi.org/10.1097/AOG.0b013e3181c33c72

7. Johnson N, Barlo, D, Lethaby A, et al. Methods of hysterectomy: systematic review and metha-analysis of randomized controlled trials. BMJ 2005; 330: 1478; http://dx.doi.org/10.1136/ bmj.330.7506.1478

8. American College of Obstetricians and Gynecologists. ACOG Committee Opinion. Number 311, April 2005. Appropriate use of laparoscopically assisted vaginal hysterectomy. Obstet Gynecol 2005; 105: 929

9. Tozzi R, Malur S, Koehler C, et al. Analysis of morbidity in patients with endometrial cancer: is there a commitment to offer laparoscopy? Gynecol Oncol 2005; 97: 4-9; http://dx.doi.org/10.1016/j. ygyno.2004.12.048

10. Ribeiro SC, Ribeiro RM, Santos NC, et al. A randomized study of total abdominal, vaginal and laparoscopic hysterectomy. Int J Gynaecol Obstet 2003; 83: 37-43; http://dx.doi.org/10.1016/S00207292(03)00271-6

11. Pelosi MA 2nd, Pelosi MA 3rd. Pelosi minilaparotomy hysterectomy: a non-endoscopic minimally invasive alternative to laparoscopy and laparotomy. Surg Technol Int 2004; 13: 57-167

12. Sheth S. Vaginal or abdominal hysterectomy. In: Sheth S, Sutton CJG (Eds). Menorrhagia. Oxford: Isis Medical Media, 1999; p. 213

13. Reich, H. Advanced laparoscopic techniques. In: Sutton, CJG, Diamond, MP (Eds). Endoscopic Surgery for Gynaecologists, 2nd ed. London: WB Saunders, 1998; p. 54

14. Kho RM, Hilger WS, Hentz JG, et al. Robotic hysterectomy: technique and initial outcomes. Am J Obstet Gynecol 2007; 197: 113.e1-4

15. Beste TM, Nelson KH, Daucher JA. Total laparoscopic hysterectomy utilizing a robotic surgical system. JSLS 2005; 9: 13-5

16. Fanfani F, Fagotti A, Scambia G. Laparoendoscopic single-site surgery for total hysterectomy. Int J Gynaecol Obstet 2010; 109: 76-7; http://dx.doi.org/10.1016/j.ijgo.2009.10.010

17. Possover M, Krause N, Plaul K, et al. Laparoscopic para-aortic and pelvic lymphadenectomy: experience with 150 patients and review of the literature. Gynecol Oncol 1998; 71: 19-28; http:// dx.doi.org/10.1006/gyno.1998.5107

18. Altgassen C, Michels W, Schneider A. Learning laparoscopic-assisted hysterectomy. Obstet Gynecol 2004; 104: 308; http://dx.doi.org/10.1097/01.AOG.0000132806.46344.05

19. Wattiez A, Cohen SB, Selvaggi L. Laparoscopic hysterectomy. Curr Opin Obstet Gynecol 2002; 14: 417-22; http://dx.doi.org/10.1097/00001703-200208000-00009

20. Winer WK, Lyons TL. Suggested set-up and layout of instruments and equipment for advanced operative laparoscopy. J Am Assoc Gynecol Laparosc 1995; 2: 231-4; http://dx.doi.org/10.1016/ S1074-3804(05)80025-8

21. Lyons T, Falcone T, Falk SJ. Laparoscopic approach to hysterectomy. Available at: http://www.uptodate.com/contents/laparoscopic-approach-to-hysterectomy. Last accessed december, 2012

22. Massarweh NN, Cosgriff N, Slakey DP. Electrosurgery: history, principles, and current and future uses. J Am Coll Surg 2006; 202: 520-30; http://dx.doi.org/10.1016/j.jamcollsurg.2005.11.017

23. Tucker RD. Laparoscopic electrosurgical injuries: survey results and their implications. Surg Laparosc Endosc 1995; 5: 311-7 
24. Nazik H, Bodur S, Api M, et al. Glutaraldehyde-induced bowel injury during gynecologic laparoscopy. J Minim Invasive Gynecol 2012; 19: 756-7; http://dx.doi.org/10.1016/j.jmig.2012.08.002

25. Sutton PA, Awad S, Perkins AC, et al. Comparison of lateral thermal spread using monopolar and bipolar diathermy, the Harmonic Scalpel and the Ligasure. Br J Surg 2010; 97: 428-33; http:// dx.doi.org/10.1002/bjs.6901

26. Erian J, El-Toukhy T, Chandakas S, et al. One hundred cases of laparoscopic subtotal hysterectomy using the PK and Lap Loop systems. J Minim Invasive Gynecol 2005; 12: 365-9; http://dx.doi. org/10.1016/j.jmig.2005.05.007

27. Ahmad G, Duffy JM, Phillips K, et al. Laparoscopic entry techniques. Cochrane Database Syst Rev 2008; (2): CD006583; doi: 10.1002/14651858.CD006583

28. McCall ML. Posterior culdeplasty; surgical correction of enterocele during vaginal hysterectomy; a preliminary report. Obstet Gynecol 1957; 10: 595; http://dx.doi.org/10.1097/00006250-19571200000001

29. Makai G, Isaacson K. Complications of gynecologic laparoscopy. Clin Obstet Gynecol 2009; 53: 401-11; http://dx.doi.org/10.1097/GRF.0b013e3181b0c080

30. Magrina JF. Complications of laparoscopic surgery. Clin Obstet Gynecol 2002; 12: 16-24 